\title{
Overview of deeply virtual Compton scattering
}

\section{A.V. Belitsky*}

Department of Physics, University of Maryland, MD 20742-4111, College Park, USA

E-mail: 'belitsky@physics.umd.edư

D. Müller ${ }^{\dagger}$

Fachbereich Physik, Universität Wuppertal, D-42097 Wuppertal, Germany

E-mail: 'dmuelier@theorie.physik. uni-wuppertal.dè

ABStRACT: We give a short overview of recent developments in understanding of the deeply virtual Compton scattering on the proton target.

\section{Introduction}

Deeply virtual Compton scattering (DVCS), recently measured by HERMES [i]1], H1 [2]-1] and CLAS [3.]. collaborations at DESY and Jefferson Lab, is the cleanest hadronic reaction that gives access to the generalized parton distributions (GPDs) [治]. GPDs are the probability amplitudes to knock out a parton from, say, the nucleon and to put it back with a different longitudinal momentum fraction. The probed partonic state is characterized, of course, by quantum numbers, like flavor, spin, etc. GPDs unify a number of known concepts of hadronic physics: they are related to parton densities, wave functions, and parton form factors, coupled also to higher spin probes. Recall that conventional form factors are encoded in matrix elements of local currents without derivatives, while for the case at hand, operators contain any number $n$ of derivatives. For $n=1$ the form factors carry information on the orbital angular momentum carried by constituents in the proton, see the second paper of Ref. [魚. Apart from DVCS and other related two-photon processes, GPDs also contribute to the hard leptoproduction of mesons, however, their theoretical description involves a new unknown nonperturbative input, a meson distributions amplitude, which complicates the disentanglement of GPDs from measurements.

\section{Differential cross section of $e N \rightarrow e^{\prime} N^{\prime} \gamma$}

Presently, we concentrate on the structure of the cross section for electroproduction of the real photon off the proton $e(k) N\left(P_{1}\right) \rightarrow e\left(k^{\prime}\right) N\left(P_{2}\right) \gamma\left(q_{2}\right)$, whose amplitude is the sum of

${ }^{*}$ This work, UMD-PP\#02-018, was supported by the US DOE under contract DE-FG02-93ER40762.

${ }^{\dagger}$ Speaker. 
Bethe-Heitler (BH) and the wanted DVCS amplitude. The four-fold cross section

$$
\frac{d \sigma}{d x_{\mathrm{B}} d y d\left|\Delta^{2}\right| d \phi}=\frac{\alpha^{3} x_{\mathrm{B}} y}{8 \pi \mathcal{Q}^{2}}\left(1+\frac{4 M^{2} x_{\mathrm{B}}^{2}}{\mathcal{Q}^{2}}\right)^{-1 / 2}\left|\frac{\mathcal{T}_{\mathrm{BH}}+\mathcal{T}_{\mathrm{DVCS}}}{e^{3}}\right|^{2},
$$

depends on the Bjorken variable $x_{\mathrm{B}}=\mathcal{Q}^{2} /\left(2 P_{1} \cdot q_{1}\right)$, the squared $t$-channel momentum transfer $\Delta^{2}=\left(P_{2}-P_{1}\right)^{2}$, the lepton energy loss $y=P_{1} \cdot q_{1} / P_{1} \cdot k$, and the azimuthal angle $\phi$, see Fig. ${ }_{11}^{1}$. The resolution scale $\mathcal{Q}^{2}=-q_{1}^{2}$ is given by the virtuality of the incoming photon. In our frame, the angular dependence of separate components in the cross section $\left(\overline{2} \cdot \bar{l}_{1}\right)$ is given by a finite sum of Fourier harmonics:

$$
\begin{aligned}
& \left|\mathcal{T}_{\mathrm{BH}}\right|^{2}=\frac{e^{6}}{x_{\mathrm{B}}^{2} y^{2} \Delta^{2} \mathcal{P}_{1}(\phi) \mathcal{P}_{2}(\phi)}\left\{c_{0}^{\mathrm{BH}}+\sum_{n=1}^{2}\left[c_{n}^{\mathrm{BH}} \cos (n \phi)+s_{n}^{\mathrm{BH}} \sin (n \phi)\right]\right\} \\
& \left|\mathcal{T}_{\mathrm{DVCS}}\right|^{2}=\frac{e^{6}}{y^{2} \mathcal{Q}^{2}}\left\{c_{0}^{\mathrm{DVCS}}+\sum_{n=1}^{2}\left[c_{n}^{\mathrm{DVCS}} \cos (n \phi)+s_{n}^{\mathrm{DVCS}} \sin (n \phi)\right]\right\} \\
& \mathcal{T}_{\mathrm{DVCS}} \mathcal{T}_{\mathrm{BH}}^{*}+\mathcal{T}_{\mathrm{DVCS}}^{*} \mathcal{T}_{\mathrm{BH}}=\frac{ \pm e^{6}}{x_{\mathrm{B}} y^{3} \mathcal{P}_{1}(\phi) \mathcal{P}_{2}(\phi) \Delta^{2}}\left\{c_{0}^{\mathcal{I}}+\sum_{n=1}^{3}\left[c_{n}^{\mathcal{I}} \cos (n \phi)+s_{n}^{\mathcal{I}} \sin (n \phi)\right]\right\} .
\end{aligned}
$$

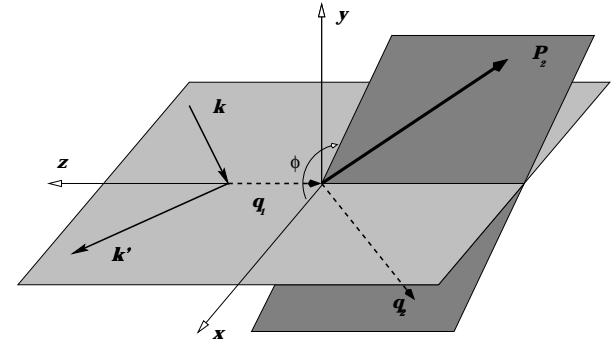

Figure 1: The kinematics for leptoproduction in the target rest frame, where the azimuthal of the proton momentum with respect to the lepton scattering plane is $\phi$.

Here the $+(-)$ sign stands for electron (positron) beam. In the squared $\mathrm{BH}$ and the interference term an additional $\phi$ dependence arises from the scaled lepton $\mathrm{BH}$ propagators $\mathcal{P}_{i}(\phi)=A_{i}+B_{i} \cos (\phi)$, which can be rather strong for large $y$. The coefficients $c_{n}$ and $s_{n}$ depend on the kinematical variables $y, x_{\mathrm{B}}$, $\Delta^{2}, \mathcal{Q}^{2}$, and lepton and hadron polarizations.

The Fourier coefficients (FCs) $c_{1}^{\mathcal{I}}$ and $s_{1}^{\mathcal{I}}$ $\left[c_{0}^{\mathcal{I}}, c_{2}^{\mathcal{I}}\right.$, and $\left.s_{2}^{\mathcal{I}}\right]$ as well as $c_{0}^{\text {DVCS }}\left[c_{1}^{\text {DVCS }}\right.$, and $s_{1}^{\text {DVCS}}$ appear at the twist-two [-three] level, while even higher harmonics are suppressed by powers of $\alpha_{s}$ or $1 / \mathcal{Q}^{2}$. For an unpolarized target we have in the twist-two sector

$$
\begin{aligned}
c_{0, \text { unp }}^{\text {DVCS }} & =2\left(2-2 y+y^{2}\right) \mathcal{C}_{\text {unp }}^{\text {DVCS }}\left(\mathcal{F}, \mathcal{F}^{*}\right) \\
\left\{\begin{array}{c}
c_{1, \text { unp }}^{\mathcal{I}} \\
s_{1, \text { unp }}^{\mathcal{I}}
\end{array}\right\} & =8 K\left\{\begin{array}{c}
-\left(2-2 y+y^{2}\right) \\
\lambda y(2-y)
\end{array}\right\}\left\{\begin{array}{l}
\operatorname{Re} \\
\operatorname{Im}
\end{array}\right\} \mathcal{C}_{\text {unp }}^{\mathcal{I}}(\mathcal{F})
\end{aligned}
$$

where $K \approx \sqrt{\left(1-x_{\mathrm{B}}\right)(1-y)} \Delta_{\perp} / \mathcal{Q}$ and $\lambda$ is the lepton helicity. The $\mathcal{C}$ s are functions of the so-called Compton form factors $(\mathrm{CFFs}) \mathcal{F}=\{\mathcal{H}, \mathcal{E}, \widetilde{\mathcal{H}}, \widetilde{\mathcal{E}}\}$ which parametrize the DVCS tensor and they have been worked out in Refs.

$$
\begin{aligned}
& \mathcal{C}_{\text {unp }}^{\mathcal{I}}=F_{1} \mathcal{H}+\frac{x_{\mathrm{B}}}{2-x_{\mathrm{B}}}\left(F_{1}+F_{2}\right) \widetilde{\mathcal{H}}-\frac{\Delta^{2}}{4 M^{2}} F_{2} \mathcal{E}, \quad \mathcal{C}_{\text {unp }}^{\text {DVCS }}=\frac{1}{\left(2-x_{\mathrm{B}}\right)^{2}}\left\{4\left(1-x_{\mathrm{B}}\right)|\mathcal{H}|^{2}\right. \\
& \left.+4\left(1-x_{\mathrm{B}}\right)|\widetilde{\mathcal{H}}|^{2}-2 x_{\mathrm{B}}^{2} \Re \mathrm{e}\left(\mathcal{H} \mathcal{E}^{*}+\widetilde{\mathcal{H}} \widetilde{\mathcal{E}}^{*}\right)-\left(x_{\mathrm{B}}^{2}+\left(2-x_{\mathrm{B}}\right)^{2} \frac{\Delta^{2}}{4 M^{2}}\right)|\mathcal{E}|^{2}-x_{\mathrm{B}}^{2} \frac{\Delta^{2}}{4 M^{2}}|\widetilde{\mathcal{E}}|^{2}\right\},
\end{aligned}
$$


where $F_{1}$ and $F_{2}$ are the nucleon Dirac and Pauli form factors, respectively. The twist-three

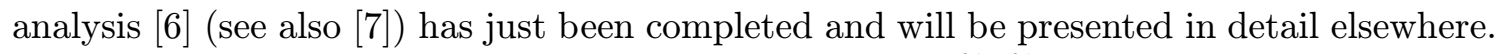
The CFFs are expressed in terms of GPDs $F=\{H, E, \widetilde{H}, \widetilde{E}\}$. At leading order (LO) of perturbation theory the relations read

$$
\left\{\begin{array}{c}
\mathcal{H} \\
\widetilde{\mathcal{H}}
\end{array}\right\}\left(\xi, \Delta^{2}, \mathcal{Q}^{2}\right)=\sum_{i=u, d, s} \int_{-1}^{1} \frac{d x}{\xi}\left(\frac{Q_{i}^{2}}{1-x / \xi-i 0} \mp\{\xi \rightarrow-\xi\}\right)\left\{\begin{array}{c}
H_{i} \\
\widetilde{H}_{i}
\end{array}\right\}\left(x, \xi, \Delta^{2}, \mathcal{Q}^{2}\right)
$$

where $Q_{i}$ is the fractional quark charge and $\xi=x_{\mathrm{B}} /\left(2-x_{\mathrm{B}}\right)$. Analogous formulae are valid for the spin-flip CFFs $\mathcal{E}$ and $\widetilde{\mathcal{E}}$. Unfortunately, from Eq. ( $(\overline{2} . \overline{5})$ one can not practically deconvolute GPDs. However, as Eq. (2.2.4) demonstrates, those observables which are sensitive to $s_{1}^{\mathcal{I}}$ allow to measure directly GPDs (at LO) for $x= \pm \xi$. Generally, one has to rely on models with a set of free parameters, which have to be adjusted to experimental data.

\section{Observables and extraction of model parameters}

For the low- $\mathcal{Q}^{2}$ kinematical settings of the present experiments $\left[\begin{array}{l}1 \\ 1\end{array}, \overline{3}\right]$ in is desirable to separate the twist-two and -tree sector, since a priori the latter can contaminate the former. This procedure is practically possible only with facilities where positive and negative lepton beams are available by means of charge asymmetries and Fourier analysis.

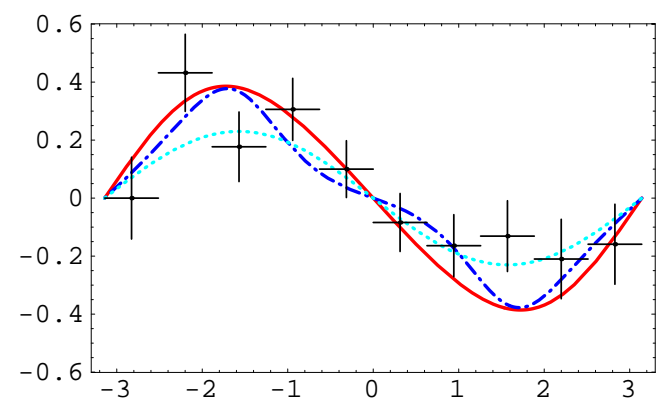

Figure 2: $\quad A_{\mathrm{LU}}$ as a function of $\phi_{\gamma}^{\prime}=$ $\pi-\phi$ at HERMES versus model predictions: $\mathrm{A}_{W W}$ (solid) and $\mathrm{B}_{W W+q G q}$ (dash-dotted).

The charge-odd combinations of cross section (י-1) gives the interference term whose coefficients can be extracted by the weighting procedure,

$$
\left\{\begin{array}{l}
c_{n}^{\mathcal{I}} \\
s_{n}^{\mathcal{I}}
\end{array}\right\} \propto \int_{0}^{2 \pi} d w\left\{\begin{array}{l}
\cos \\
\sin
\end{array}\right\}(n \phi)\left(\frac{d^{+} \sigma}{d \phi}-\frac{d^{-} \sigma}{d \phi}\right)
$$

with the measure $d w \propto \mathcal{P}_{1}(\phi) \mathcal{P}_{2}(\phi) d \phi$. The FCs of the squared DVCS term can be obtained from the charge-even part after subtraction of the squared $\mathrm{BH}$ term, i.e. $d^{\mathrm{DVCS}} \sigma=\left(d \sigma^{+}+\right.$ $\left.d^{-} \sigma\right) / 2-d^{\mathrm{BH}} \sigma$, and weighting with respect to the measure $d \phi$. Asymmetries are normalized with respect to $\int_{0}^{2 \pi} d \phi \frac{d^{+} \sigma+d^{-} \sigma}{d \phi}$, which does not contain twist-three corrections. Unfortunately, for single-charge lepton beam machines this clear separation of twist-two and -three effects cannot be achieved - also not with spin or azimuthal asymmetries. For instance,

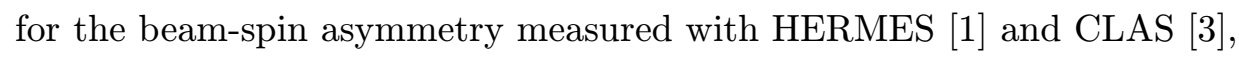

$$
A_{\mathrm{LU}}(\phi)=\left(d \sigma^{\uparrow}-d \sigma^{\downarrow}\right) /\left(d \sigma^{\uparrow}+d \sigma^{\downarrow}\right)
$$

the leading twist term $s_{1}^{\mathcal{I}}$ in the numerator will be affected by $s_{2}^{\mathcal{I}}$ and $s_{1}^{\text {DVCS }}$ while the denominator will be the sum of all three contributions in Eq. (2.2i). Fortunately, if the condition $(1-y) \Delta^{2} / y^{2} \mathcal{Q}^{2} \ll 1$ is fulfilled, $s_{1}^{\text {DVCS }}$ is kinematically suppressed and, moreover, the squared $\mathrm{BH}$ term $c_{0}^{\mathrm{BH}}$ dominates in the denominator. Thus, the lepton $\mathrm{BH}$ propagators approximately cancel in Eq. $\left(\overline{3}_{2}^{2} \cdot \overline{2}\right)$ and $A_{\mathrm{LU}}(\phi) \approx \pm\left(x_{\mathrm{B}} s_{1}^{\mathcal{I}} / y c_{0}^{\mathrm{BH}}\right) \sin (\phi)$. 
Since the number of experimental observables is very limited we are confined to use GPD models in order to confront theoretical expectations with data. Let us briefly outline, however, a way how model parameters can be fit to experiments in particular kinematical situations. An ansatz is based on a simplistic factorization of $\Delta^{2}$ and $(x, \xi)$ dependence. Sum rules and reduction formulae and ad hoc assumptions result into a model for, e.g., $H_{i}$

$$
H_{i}=F_{1}^{i}\left(\Delta^{2}\right) \int_{0}^{1} d y \int_{-1+y}^{1-y} d z \delta(y+\xi z-x) q_{i}(y) N\left(b_{i}\right) \frac{\left[(1-y)^{2}-z^{2}\right]^{b_{i}}}{(1-y)^{2 b_{i}+1}}+D_{i}
$$

where $q_{i}$ are the parton densities given at the input scale $\mathcal{Q}_{0}$ and $N\left(b_{i}\right)=\frac{\Gamma\left(b_{i}+3 / 2\right)}{\sqrt{\pi} \Gamma\left(b_{i}+1\right)}$. For valence quarks the form factors are fixed by the sum rules, while for sea quarks we parametrize them as $F_{1}^{\text {sea }}\left(\Delta^{2}\right)=\left(1-B_{\text {sea }} \Delta^{2} / 3\right)^{-3}$ with a free slope parameter $B_{\text {sea }}$. The

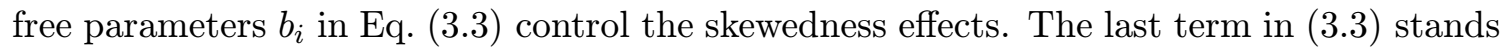
for the mesonic-like contributions, see $\left[\bar{i} \bar{i}_{1}\right]$. Parametrizations for the spin-flip GPDs are obtained by the replacements: $F_{1}^{i} \rightarrow F_{2}^{i}$ and $D_{i} \rightarrow-D_{i} . \widetilde{H}_{i}$ is given in terms of polarized parton densities, while $\widetilde{E}_{i}$ is dominated by the pion pole, see $\left[\bar{T}_{i}\right]$.

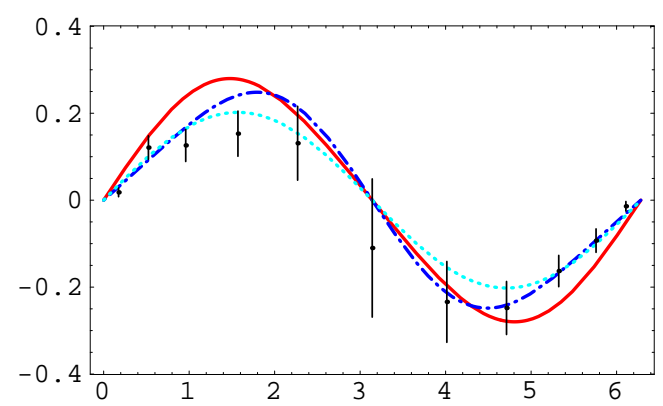

Figure 3: $A_{\mathrm{LU}}$ as a function of $\phi_{\gamma}^{\prime}=\pi-$ $\phi$ at CLAS versus model predictions. Same assignments as in Fig. $\overline{2}_{r}^{\prime}$

Assuming the MRS $\mathrm{A}^{\prime}$ and GS A densities at the input scale $\mathcal{Q}_{0}^{2}=4 \mathrm{GeV}^{2}$, we analyzed the existing DVCS data with these models at LO in the twist-three approximation with neglected $\mathcal{Q}^{2}$-evolution. The unpolarized cross section at small $x_{\mathrm{B}}$ as measured by $\mathrm{H} 1$ [2]] is dominated by $\mathcal{H}_{\text {sea }}$, i.e., $\mathcal{C}_{\text {unp }}^{\text {DVCS }} \approx|\mathcal{H}|^{2}$ for $-\Delta^{2} \ll 4 M^{2}$. We found that the skewedness effect is small and the fall-off with $\Delta^{2}$ is strong. The choice $b_{\text {sea }} \rightarrow \infty$ and $B_{\text {sea }}=9 \mathrm{GeV}^{2}$ gives a good agreement with the data. Using $b_{\text {val }}$ and sea-quark magnetic moment $\kappa_{\text {sea }}$ as free parameters we distinguish two models: A with $b_{\text {val }}=1$ and $\kappa_{\text {sea }}=0$, and B with $b_{\text {val }} \rightarrow \infty$ and $\kappa_{\text {sea }}=-3$.

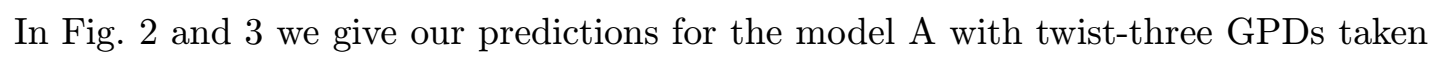
in the Wandzura-Wilczek approximation (solid line) [6] and the model B with quarkgluon-quark correlations accounted for in the twist-three GPDs (dash-dotted line) versus experimental data. For HERMES and CLAS plots, the dotted line denotes the $\sin \phi$ with the amplitudes 0.23 and 0.202 , respectively, fit to the data by the collaborations. At HERMES the integrated beam-spin asymmetry $A_{\mathrm{LU}}=-0.23 \pm 0.04$ (stat) \pm 0.03 (syst) has been measured at the average values $\left\langle\mathcal{Q}^{2}\right\rangle=2.6 \mathrm{GeV}^{2},\left\langle x_{\mathrm{B}}\right\rangle=0.11$ and $\left\langle-\Delta^{2}\right\rangle=0.27 \mathrm{GeV}^{2}$ [i]1. Integrating our predictions $\mathrm{A}_{W W}$ (solid) and $\mathrm{B}_{W W+q G q}$ (dash-dotted) from Fig. 12i we have $A_{\mathrm{LU}}=-0.27$ and $A_{\mathrm{LU}}=-0.16$, respectively. Both of them are compatible with the data. The beam-spin asymmetry as measured by CLAS collaboration was integrated over the region: $1 \mathrm{GeV}^{2}<\mathcal{Q}^{2}<1.75 \mathrm{GeV}^{2}, 0.13<x_{\mathrm{B}}<0.35$, and $0.1 \mathrm{GeV}^{2}<-\Delta^{2}<0.3 \mathrm{GeV}^{2}$ with $W>2 \mathrm{GeV}$. Their integrated result is fit by $A_{\mathrm{LU}}(\phi)=\alpha \sin (\phi)+\beta \sin (2 \phi)$ with

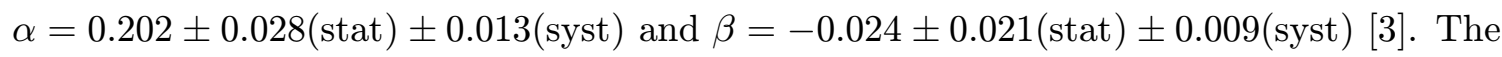
model $\mathrm{A}_{W W}$ fails to describe the data resulting into $\alpha=0.28$ and $\beta=0.014$. However for the model $\mathrm{B}_{W W+q G q}$ we find $\alpha=0.24$ and $\beta=-0.03$ in fairly good agreement with the 
measurement. Note that contrary to $\alpha$, the second harmonic parametrized by $\beta$ is very sensitive to quark-gluon-quark correlations.

\section{Beyond leading order and power}

So far we have shown that the DVCS results measured in three different experiments are consistent for a set of GPD models with DVCS amplitudes evaluated at LO within the twist-three approximation. Once we will have a rough idea on the magnitude of model parameters a refined analysis has to be conducted taking into account higher order effects in QCD coupling constant. In the twist-two sector, they are completely worked out at NLO [8] large radiative corrections can be generated by certain models of gluon GPDs. The latter are induced by strong evolution effects and, thus, these models can be experimentally tested in the small- $x_{\mathrm{B}}$ region. The gluonic contributions can be set to zero by an appropriate choice of the factorization scale. In this scheme we have moderate $20 \%$ corrections to the CFFs, however, the DVCS cross section then gets corrected by $40 \%$.

As we have observed the CLAS result presumably indicates a non-negligible contribution from multiparton correlations in the nucleon. Therefore, a further study of higher twist effects is necessary. Since $\mathcal{Q}^{2}$ is of the order of one $\mathrm{GeV}^{2}$, one expects also a sizable contamination by target mass $M^{2} / \mathcal{Q}^{2}$ effects. A first step in this direction has been made in $[\overline{9}]$, however, a complete treatment requires a consideration of mass effects stemming form multiparticle operators.

\section{References}

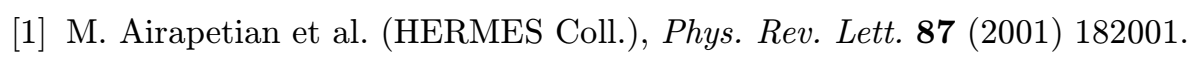

[2] C. Adloff et al. (H1 Coll.), 'Ph

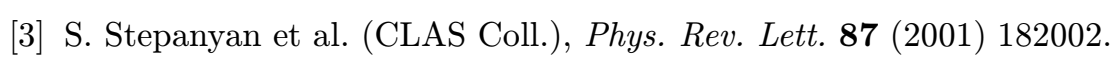

[4] D. Müller, D. Robaschik, B. Geyer, F.M. Dittes, J. Hořejši, Fortschr. Phys. 42 2 (1994) 101;

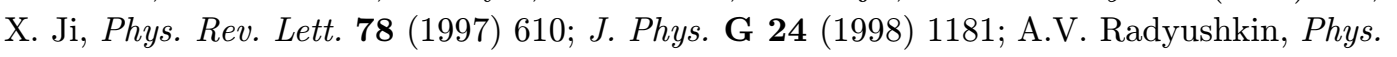
L

[5] M. Diehl, T. Gousset, B. Pire, J.P. Ralston, Phys. Lett. B 411 (1997) $193 \overline{3}$ A.V. Belitsky, D. Müller, L. Niedermeier, A. Schäfer, 'Nucl. Phys. B $\mathbf{5 9 3}(2001) 289$.

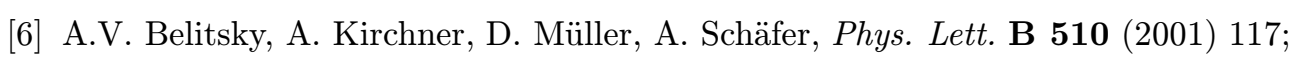
A.V. Belitsky, A. Kirchner, D. Müller, hep-ph/0106228 UMD-PP\#02-011 (in preparation).

[7] N. Kivel, M.V. Polyakov, M. Vanderhaeghen, $\overline{P h y s}$ Rev. $\mathbf{D} \mathbf{6} \overline{3}(\overline{2001)} \overline{1} \overline{4} \overline{0} \overline{1} \bar{r}$ K. Goeke, M.V. Polyakov, M. Vanderhaeghen, 'Prog. Part. Nucl. Phys. 47 (2001) 401

[8] A.V. Belitsky, D. Müller, 'Nucl.Phys.

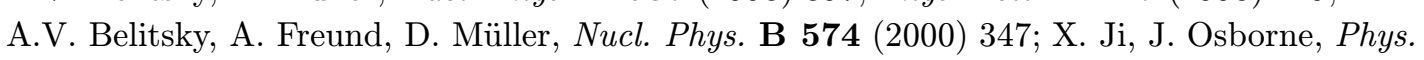
Rev. $\overline{5} \overline{5}(\overline{1} \overline{9} 8) 0940 \overline{1} \overline{8}_{i}$ L. Mankiewicz, G. Piller, E. Stein, M. Vänttinen, T. Weigl, 'Ph Lett. B $425(1998) 186$.

[9] A.V. Belitsky, D. Müller, Physs. Lett. $\bar{B}^{-} \overline{5} \overline{0} \overline{7}\left(200 \overline{1} \overline{1} \overline{1} \overline{3} \bar{r}_{\mathrm{r}}^{\prime}\right.$ 